\title{
Subspace Perturbation Bounds with an Application to Angle of Arrival Estimation using the MUSIC Algorithm
}

\author{
Connor Delaosa $^{1}$, Jennifer Pestana ${ }^{2}$, Stephan Weiss ${ }^{1}$, Ian K. Proudler ${ }^{1}$ \\ ${ }^{1}$ Dept. Electronic \& Electrical Engineering, University of Strathclyde, Glasgow G1 1XW, Scotland \\ ${ }^{2}$ Dept. Mathematics \& Statistics, University of Strathclyde, Glasgow G1 1XH, Scotland \\ \{connor.delaosa,jennifer.pestana,stephan.weiss,ian.proudler\}@strath.ac.uk
}

\begin{abstract}
This paper explores how angle of arrival (AoA) estimation using the multiple signal classification (MUSIC) algorithm is affected by estimation errors in the space-time covariance matrix. In particular, we explore how this estimation error perturbs the signal-plus-noise and noise-only subspaces of the matrix, and how this subsequently affects the performance of MUSIC for AoA estimation. This subspace perturbation is shown to depend on the space-time covariance matrix itself, the sample size over which it is estimated, as well as the distance of the smallest signal-related eigenvalue to the noise floor. We link a bound on this perturbation to a bound on MUSIC performance, and demonstrate its utility for AoA estimation in simulations.

Index Terms-space-time covariance matrix, parahermitian matrix, cross-correlation sequence, estimation, angle of arrival, MUSIC
\end{abstract}

\section{INTRODUCTION}

In broadband array processing a space-time covariance matrix forms the basis for describing the second order statistics of the data recorded from $M$ sensor measurements. These measurements, $\mathbf{x}[n] \in \mathbb{C}^{M}$, can be used to calculate the space-time covariance matrix matrix as $\mathbf{R}[\tau]=\mathcal{E}\left\{\mathbf{x}[n] \mathbf{x}^{\mathrm{H}}[n-\tau]\right\} \in$ $\mathbb{C}^{M \times M}$, where $\mathcal{E}\{\cdot\}$ is the expectation operator and $\{\cdot\}^{\mathrm{H}}$ is the Hermitian transpose operator. In narrowband signal processing, which captures the phase shifts between sensor elements. it suffices to use the instantaneous covariance matrix $\mathbf{R}[0]$ only. If we move to the broadband domain then explicit time delay information must be preserved, and requires the inclusion of the lag component $\tau$ into the space-time covariance matrix $\mathbf{R}[\tau]$, which contains auto and -cross-correlation sequences. This space-time covariance matrix may need to be estimated such as in the situations of limited availability of data samples or when data must be restricted to a small time frame to assume stationarity. The estimation and the resulting perturbations of this matrix has been explored in the past [1] [4].

In the broadband case, the multiple signal classification (MUSIC) algorithm [5] uses the space-time covariance matrix to estimate the angle of arrival (AoA) of a particular source. If these matrices are estimated from finite data, we can use

This work was supported in parts by the Engineering and Physical Sciences Research Council (EPSRC) Grant number EP/S000631/1 and the MOD University Defence Research Collaboration in Signal Processing, Dstl, and a John Anderson Research Award by the University of Strathclyde. perturbation theory to gain a measure of how much this result is perturbed. Therefore, an understanding of how this algorithm performs under estimation is important. From [2], [6] we know the variance of the estimation error, and subsequently how eigenvalues and eigenspaces are perturbed as a result of the sample size, $N$. In this paper, we are concerned with how this perturbation of $\mathbf{R}[\tau]$ impacts the MUSIC algorithm and in particular how the algorithm degrades as a function of $N$.

In the past, papers have looked at the noise and signal subspaces crucial for the performance of MUSIC. In particular, [7], [8] show that when using the singular value decomposition (SVD) and the root-MUSIC algorithm, respectively, subspace methods for a small fixed sample size severely degrade as the signal-to-noise ratio (SNR) decreases greatly. In order to improve the direction of arrival (DoA) estimates for subspace methods in poor conditions, various papers modify the MUSIC algorithm in different ways such as introducing: a new estimator [9], an innovative MUSIC algorithm to decrease the threshold of SNR before degradation [10], or an iterative algorithm to reduce unwanted terms in the sample spatially correlated covariance matrix [11]. Under the influence of varying modelling errors [12] investigates the MUSIC estimator showing its performance degradation for higher error terms. In contrast, in this paper we look to extend [7], [8] to the broadband case where we use an eigenvalue decomposition (EVD) to obtain the subspaces as we vary $N$. We apply perturbation theory in order to understand the degradation of the traditional MUSIC algorithm for a fixed SNR and a range of sample sizes with the aim of establishing a link between a bound of this perturbation and a bound on the performance of MUSIC.

This paper is laid out as follows; in Sec. II the data model and the MUSIC algorithm based on ideal quantities are stated. Sec. III demonstrates the construction of an estimated spacetime covariance matrix, the estimation error between this and the ideal case and how this error can lead to eigenvalues and eigenspaces being perturbed. In Sec. IV we relate the perturbation of subspaces to the MUSIC algorithm estimated from limited data and therefore derive a bound to characterise this relationship. We then, in Sec. V, verify the theory through simulation and test the performance of the bound and related 
quantities.

\section{Angle of ARrival Estimation}

\section{A. Data Model}

Let $\mathbf{x}[n] \in \mathbb{C}^{M}$ be a data vector recorded from $M$ sensor measurements $x_{m}[n], m=1, \ldots, M$. This array is illuminated by $K$ sources in the far-field, and while we assume no loss in gain, these source signals $s_{k}[n], k=1, \ldots, K$, arrive at the different sensors with time delays $\tau_{k, m}$. Therefore, the propagation environment can be described by a broadband steering vector $\mathbf{a}_{k}[n]$,

$$
\mathbf{a}_{k}[n]=\frac{1}{\sqrt{M}}\left[\begin{array}{c}
f\left[n-\tau_{k, 1}\right] \\
\vdots \\
f\left[n-\tau_{k, M}\right]
\end{array}\right],
$$

with $f[n-\tau]$ implementing a fractional delay filter [13], [14]. For the time delay $\tau_{k, m}=\frac{1}{c T_{\mathrm{s}}} \mathbf{k}_{k}^{\mathrm{T}} \mathbf{r}_{m}, c$ denotes the propagation speed in the medium, $T_{\mathrm{s}}$ is the sampling period, $\mathbf{k}_{k}$ is the normal vector to the wavefront of the $k$ th source signal and $\mathbf{r}_{m}$ is the location of the $m$ th sensor in three-dimensional space. Using the steering vector, a model for the received data is

$$
\mathbf{x}[n]=\sum_{k=1}^{K} \mathbf{a}_{k}[n] s_{k}[n]+\mathbf{v}[n]
$$

where $\mathbf{v}[n]$ is spatially and temporally uncorrelated Gaussian noise with covariance $\mathcal{E}\left\{\mathbf{v}[n] \mathbf{v}^{\mathrm{H}}[n-\tau]\right\}=\sigma_{v}^{2} \mathbf{I} \delta[\tau]$ and $\mathbf{I}$ is an $M \times M$ identity matrix.

The cross-spectral density (CSD) matrix, $\boldsymbol{R}(z)$, originates from the $z$-transform of $\mathbf{R}[\tau]$ i.e. $\boldsymbol{R}(z)=\sum_{\tau} \mathbf{R}[\tau] z^{-\tau}$, or in short $\boldsymbol{R}(z) \bullet-\circ \mathbf{R}[\tau]$, where $\bullet-\circ$ denotes a transform pair. In almost all cases $\boldsymbol{R}(z)$ admits a parahermitian matrix EVD (PhEVD) [15], [16] such that

$$
\boldsymbol{R}(z)=\boldsymbol{Q}(z) \boldsymbol{\Lambda}(z) \boldsymbol{Q}^{\mathrm{P}}(z) .
$$

Here $\boldsymbol{Q}(z) \in \mathbb{C}^{M \times M}$ is paraunitary i.e. $\boldsymbol{Q}(z) \boldsymbol{Q}^{\mathrm{P}}(z)=\boldsymbol{I}$ where $\{\cdot\}^{\mathrm{P}}$ denotes the parahermitian operator of a given matrix such that $\boldsymbol{R}(z)=\boldsymbol{R}^{\mathrm{P}}(z)=\boldsymbol{R}^{\mathrm{H}}\left(z^{-1}\right)$. The quantity $\boldsymbol{Q}(z)$ is analytic [15] in most cases [17] and contains the eigenvectors while $\boldsymbol{\Lambda}(z)=\operatorname{diag}\left\{\lambda_{1}(z), \ldots \lambda_{M}(z)\right\}$ holds the $M$ eigenvalues.

Given the time domain model in (2), the CSD matrix for this scenario is

$$
\boldsymbol{R}(z)=\sum_{k=1}^{K} \mathbf{a}_{k}(z) S_{k}(z) \mathbf{a}_{k}^{\mathrm{P}}(z)+\sigma_{v}^{2} \mathbf{I}
$$

where $S_{k}(z)$ is the power spectral density (PSD) of the $k$ th source signal $s_{k}[n]$. This signal $s_{k}[n]$ can be tied to an uncorrelated zero-mean unit-variance Gaussian signal $u_{k}[n]$ via an innovation filter [18] $b_{k}[n] \bullet-\circ B_{k}(z)$, such that $S_{k}(z)=B_{k}(z) B_{k}^{\mathrm{P}}(z)$.

\section{B. MUSIC based on Ideal Space-Time Covariance Matrices}

Since the factors in (3) are analytic in some region of convergence including the unit circle, in the subsequent analysis we restrict ourselves to evaluating on the unit circle i.e. $z=\mathrm{e}^{\mathrm{j} \Omega}$. With knowledge of the number of sources, $K$, we partition, $\left.\boldsymbol{Q}(z)\right|_{z=\mathrm{e}^{\mathrm{j} \Omega}}$ into two subspaces such that the $K$ columns of $\boldsymbol{Q}_{\mathrm{s}}\left(\mathrm{e}^{\mathrm{j} \Omega}\right)$ span the $K$-dimensional signal-plus-noise subspace, while the columns of $\boldsymbol{Q}_{\mathrm{n}}\left(\mathrm{e}^{\mathrm{j} \Omega}\right)$ form the basis for its complement, the $M-K$-dimensional noise-only subspace. Hence

$$
\begin{aligned}
\boldsymbol{R}\left(\mathrm{e}^{\mathrm{j} \Omega}\right)=\left[\boldsymbol{Q}_{\mathrm{s}}\left(\mathrm{e}^{\mathrm{j} \Omega}\right) \boldsymbol{Q}_{\mathrm{n}}\left(\mathrm{e}^{\mathrm{j} \Omega}\right)\right] & {\left[\begin{array}{cc}
\boldsymbol{\Lambda}_{\mathrm{s}}\left(\mathrm{e}^{\mathrm{j} \Omega}\right) & 0 \\
0 & \boldsymbol{\Lambda}_{\mathrm{n}}\left(\mathrm{e}^{\mathrm{j} \Omega}\right)
\end{array}\right] . } \\
& \cdot\left[\begin{array}{l}
\boldsymbol{Q}_{\mathrm{s}}^{\mathrm{H}}\left(\mathrm{e}^{\mathrm{j} \Omega}\right) \\
\boldsymbol{Q}_{\mathrm{n}}^{\mathrm{H}}\left(\mathrm{e}^{\mathrm{j} \Omega}\right)
\end{array}\right]
\end{aligned}
$$

where $\boldsymbol{\Lambda}_{\mathrm{s}}\left(\mathrm{e}^{\mathrm{j} \Omega}\right)$ contains the $K$ eigenvalues associated with the signal-plus-noise subspace, and $\Lambda_{\mathrm{n}}\left(\mathrm{e}^{\mathrm{j} \Omega}\right)$ contains the remaining $M-K$ eigenvalues.

With these quantities now defined, it can be stated that the steering vectors, $\boldsymbol{a}_{\varphi, \vartheta}\left(\mathrm{e}^{\mathrm{j} \Omega}\right)=\left[\mathbf{a}_{1}\left(\mathrm{e}^{\mathrm{j} \Omega}\right), \ldots, \mathbf{a}_{K}\left(\mathrm{e}^{\mathrm{j} \Omega}\right)\right]^{\mathrm{T}}$ for azimuth $\varphi$ and elevation $\vartheta$, contribute to the signal-plusnoise subspace, $\boldsymbol{Q}_{\mathrm{s}}\left(\mathrm{e}^{\mathrm{j} \Omega}\right)$. These therefore reside within the nullspace of the noise subspace, $\boldsymbol{Q}_{\mathrm{n}}\left(\mathrm{e}^{\mathrm{j} \Omega}\right)$, i.e. the vector $\boldsymbol{Q}_{\mathrm{n}}^{\mathrm{H}}\left(\mathrm{e}^{\mathrm{j} \Omega}\right) \mathbf{a}_{\varphi, \vartheta}\left(\mathrm{e}^{\mathrm{j} \Omega}\right)$ tends toward a zero vector if $\boldsymbol{a}_{\varphi, \vartheta}\left(\mathrm{e}^{\mathrm{j} \Omega}\right)$ is the steering vector of a source. As a result, we can scan the noise-only subspace with steering vectors determined by a range of angles $\{\varphi, \vartheta\}$ and frequencies $\Omega$ such that

$$
\xi_{\operatorname{MUSIC}}\left(\mathrm{e}^{\mathrm{j} \Omega}, \varphi, \vartheta\right)=\frac{1}{\mathbf{a}_{\varphi, \vartheta}^{\mathrm{P}}\left(\mathrm{e}^{\mathrm{j} \Omega}\right) \boldsymbol{Q}_{\mathrm{n}}\left(\mathrm{e}^{\mathrm{j} \Omega}\right) \boldsymbol{Q}_{\mathrm{n}}^{\mathrm{P}}\left(\mathrm{e}^{\mathrm{j} \Omega}\right) \mathbf{a}_{\varphi, \vartheta}\left(\mathrm{e}^{\mathrm{j} \Omega}\right)},
$$

which is also known as the ideal MUSIC spectrum [5]. In practice, this metric will be computed using estimated quantities, resulting in perturbations. The next two sections will therefore investigate the estimation errors and analyse the subspace perturbation of the noise-only subspace and its effect on MUSIC.

\section{Covariance Estimation And Subspace PERTURBATIONS}

\section{A. Space-Time Covariance Estimation}

Instead of determining $\mathbf{R}[\tau]$ via an expectation operation, in practice it has to be estimated in time over a finite window of snapshots, say $N$. Given the data vector $\mathbf{x}[n]$ for $n=0, \ldots,(N-1)$, a sample space-time covariance matrix $\hat{\mathbf{R}}[\tau]$ can be calculated e.g. via an estimator [19]

$$
\hat{\mathbf{R}}[\tau]=\left\{\begin{array}{ll}
\frac{1}{N-|\tau|} \sum_{n=0}^{N-|\tau|-1} \mathbf{x}[n+\tau] \mathbf{x}^{\mathrm{H}}[n], & \tau \geq 0 \\
\frac{1}{N-|\tau|} \sum_{n=0}^{N-|\tau|-1} \mathbf{x}[n] \mathbf{x}^{\mathrm{H}}[n-\tau], & \tau<0
\end{array} .\right.
$$

Because this estimator is unbiased, for the estimation error

$$
\mathbf{E}[\tau]=\hat{\mathbf{R}}[\tau]-\mathbf{R}[\tau]
$$


we find that its power, $\mathcal{E}\left\{\|\mathbf{E}[\tau]\|_{\mathrm{F}}^{2}\right\}$, equals the variance of $\hat{\mathbf{R}}[\tau]$, which has been shown to depend on both the ground truth $\mathbf{R}[\tau]$ and the sample size $N$ [19].

\section{B. Perturbation of Eigenspaces}

With the PhEVD $\hat{\boldsymbol{R}}(z)=\hat{\boldsymbol{Q}}(z) \hat{\boldsymbol{\Lambda}}(z) \hat{\boldsymbol{Q}}^{\mathrm{P}}(z)$ we partition the eigenvalues and eigenvectors similarly to (5). The eigenvalues in $\hat{\boldsymbol{\Lambda}}\left(\mathrm{e}^{\mathrm{j} \Omega}\right)=\operatorname{blockdiag}\left\{\hat{\boldsymbol{\Lambda}}_{\mathrm{s}}\left(\mathrm{e}^{\mathrm{j} \Omega}\right) \quad \hat{\boldsymbol{\Lambda}}_{\mathrm{n}}\left(\mathrm{e}^{\mathrm{j} \Omega}\right)\right\}$ are split into a $K$-element diagonal matrix containing the eigenvalues corresponding to the signal-plus-noise-subspace and an $M-K$ diagonal matrix of values that form the noise floor. The eigenvectors are then defined as $\hat{\boldsymbol{Q}}\left(\mathrm{e}^{\mathrm{j} \Omega}\right)=$ $\left[\hat{\boldsymbol{Q}}_{\mathrm{s}}\left(\mathrm{e}^{\mathrm{j} \Omega}\right) \hat{\boldsymbol{Q}}_{\mathrm{n}}\left(\mathrm{e}^{\mathrm{j} \Omega}\right)\right]$, such that the subspaces are given by $\hat{\mathcal{U}}_{\mathrm{s}, \mathrm{n}}\left(\mathrm{e}^{\mathrm{j} \Omega}\right)=$ range $\left\{\hat{\boldsymbol{Q}}_{\mathrm{s}, \mathrm{n}}\left(\mathrm{e}^{\mathrm{j} \Omega}\right)\right\}$, with the ideal subspaces being denoted similarly. With these quantities, we assess the difference between the ground-truth noise-only subspace $\mathcal{U}_{\mathrm{n}}\left(\mathrm{e}^{\mathrm{j} \Omega}\right)$ and its estimated version $\hat{\mathcal{U}}_{\mathrm{n}}\left(\mathrm{e}^{\mathrm{j} \Omega}\right)$ as

$$
\operatorname{dist}\left\{\hat{\mathcal{U}}_{\mathrm{n}}\left(\mathrm{e}^{\mathrm{j} \Omega}\right), \mathcal{U}_{\mathrm{n}}\left(\mathrm{e}^{\mathrm{j} \Omega}\right)\right\}=\left\|\hat{\boldsymbol{P}}_{\mathrm{n}}\left(\mathrm{e}^{\mathrm{j} \Omega}\right)-\boldsymbol{P}_{\mathrm{n}}\left(\mathrm{e}^{\mathrm{j} \Omega}\right)\right\|_{2},
$$

where $\hat{\boldsymbol{P}}_{\mathrm{n}}\left(\mathrm{e}^{\mathrm{j} \Omega}\right)=\hat{\boldsymbol{Q}}_{\mathrm{n}}\left(\mathrm{e}^{\mathrm{j} \Omega}\right) \hat{\boldsymbol{Q}}_{\mathrm{n}}^{\mathrm{H}}\left(\mathrm{e}^{\mathrm{j} \Omega}\right)$ and $\boldsymbol{P}_{\mathrm{n}}\left(\mathrm{e}^{\mathrm{j} \Omega}\right)=$ $\boldsymbol{Q}_{\mathrm{n}}\left(\mathrm{e}^{\mathrm{j} \Omega}\right) \boldsymbol{Q}_{\mathrm{n}}^{\mathrm{H}}\left(\mathrm{e}^{\mathrm{j} \Omega}\right)$ are projection operators into the noise-only subspaces. To find bounds on the eigenspace perturbations we must first define the spectral distance $\delta$ between the signalplus-noise and noise-only eigenvalues as

$$
\delta=\min _{\substack{\lambda_{1} \in \boldsymbol{\Lambda}_{\mathrm{s}}\left(\mathrm{e}^{\mathrm{j} \Omega}\right) \\ \lambda_{2} \in \boldsymbol{\Lambda}_{\mathrm{n}}\left(\mathrm{e}^{\mathrm{j} \Omega}\right)}}\left|\lambda_{1}-\lambda_{2}\right|>0 ;
$$

in other words, $\delta$ is the absolute minimum difference between the signal-plus-noise and noise-only eigenvalues.

If the estimation error [6, Sec. 7.2] satisfies

$$
\left\|\boldsymbol{E}\left(\mathrm{e}^{\mathrm{j} \Omega}\right)\right\|_{2}<\delta / 5
$$

we can state that the subspace distance is bounded as follows [2], [6]:

$$
\operatorname{dist}\left\{\hat{\mathcal{U}}_{\mathrm{n}}\left(\mathrm{e}^{\mathrm{j} \Omega_{0}}\right), \mathcal{U}_{\mathrm{n}}\left(\mathrm{e}^{\mathrm{j} \Omega_{0}}\right)\right\} \leq \frac{4}{\delta}\left\|\mathbf{E}_{\mathrm{sn}}\left(\mathrm{e}^{\mathrm{j} \Omega_{0}}\right)\right\|_{2} .
$$

Here $\mathbf{E}_{\mathrm{sn}}\left(\mathrm{e}^{\mathrm{j} \Omega_{0}}\right)$ is extracted from the following partioning of a similarity transform of the estimation error matrix $\boldsymbol{E}\left(\mathrm{e}^{\mathrm{j} \Omega_{0}}\right)=$ $\left.\boldsymbol{E}\left(\mathrm{e}^{\mathrm{j} \Omega}\right)\right|_{\mathrm{e}^{\mathrm{j} \Omega}=\mathrm{e}^{\mathrm{j} \Omega_{0}}}$ with $\boldsymbol{E}\left(\mathrm{e}^{\mathrm{j} \Omega}\right) \bullet-\mathrm{E}[\tau]$,

$$
\boldsymbol{Q}^{\mathrm{H}}\left(\mathrm{e}^{\mathrm{j} \Omega_{0}}\right) \boldsymbol{E}\left(\mathrm{e}^{\mathrm{j} \Omega_{0}}\right) \boldsymbol{Q}\left(\mathrm{e}^{\mathrm{j} \Omega_{0}}\right)=\left[\begin{array}{cc}
\underbrace{\mathbf{E}_{\mathrm{s}}\left(\mathrm{e}^{\mathrm{j} \Omega_{0}}\right)}_{K} & \underbrace{\mathbf{E}_{\mathrm{sn}}^{\mathrm{H}}\left(\mathrm{e}^{\mathrm{j} \Omega_{0}}\right)}_{M-K} \\
\underbrace{\mathrm{E} \Omega_{0}}_{M}) & \underbrace{}_{\mathbf{E}_{\mathrm{n}}\left(\mathrm{e}^{\mathrm{j} \Omega_{0}}\right)}
\end{array}\right] .
$$

From the quantities defined, we can state that the subspace perturbation depends on: the ground truth covariance matrix, $\boldsymbol{R}\left(\mathrm{e}^{\mathrm{j} \Omega}\right)$, the sample size $N$ via $\mathbf{E}_{\mathrm{sn}}\left(\mathrm{e}^{\mathrm{j} \Omega_{0}}\right)$, and the distance $\delta$ i.e. by how much the 'signal eigenvalue' differs from the noise floor. We can now use this in Sec. IV to analyse the MUSIC algorithm.

\section{MUSIC based on Sample Covariance Matrices}

Following Secs. II and III-A, where the ideal MUSIC algorithm and the estimated quantities are introduced, we formulate the estimated MUSIC cost function in Sec. IV-A below. The link between its inverse and the subspace perturbation bound is then investigated in Sec. IV-B.

\section{A. Estimation of MUSIC}

Using the noise-only subspace $\hat{\boldsymbol{Q}}_{\mathrm{n}}\left(\mathrm{e}^{\mathrm{j} \Omega_{0}}\right)$, we obtain

$$
\begin{aligned}
\hat{\xi}_{\text {MUSIC }}^{-1}\left(\mathrm{e}^{\mathrm{j} \Omega_{0}}, \varphi, \vartheta\right) & =\mathbf{a}^{\mathrm{H}}\left(\mathrm{e}^{\mathrm{j} \Omega_{0}}\right) \hat{\boldsymbol{Q}}_{\mathrm{n}}\left(\mathrm{e}^{\mathrm{j} \Omega_{0}}\right) \hat{\boldsymbol{Q}}_{\mathrm{n}}^{\mathrm{H}}\left(\mathrm{e}^{\mathrm{j} \Omega_{0}}\right) \mathbf{a}\left(\mathrm{e}^{\mathrm{j} \Omega_{0}}\right) \\
& =\left\|\hat{\boldsymbol{Q}}_{\mathrm{n}}^{\mathrm{H}}\left(\mathrm{e}^{\mathrm{j} \Omega_{0}}\right) \mathbf{a}_{\varphi, \vartheta}\left(\mathrm{e}^{\mathrm{j} \Omega_{0}}\right)\right\|_{2}^{2},
\end{aligned}
$$

which defines the MUSIC spectrum derived from estimated quantities. Ideally, (14) should be zero at the angles of arrival but, due to the use of the estimated covariance matrix, it can deviate from zero by an unknown amount. Since the MUSIC metric in (14) is based on the estimated subspaces we can now relate this to Sec. III-B and describe the relationship between $\hat{\xi}_{\text {MUSIC }}^{-1}\left(\mathrm{e}^{\mathrm{j} \Omega_{0}}, \varphi_{0}, \vartheta_{0}\right)$ and the perturbation of subspaces.

\section{B. Perturbation Bounds of the MUSIC Metric}

In the following, we analyse the inverse MUSIC spectrum in (14) in the direction of arrival of the source, $\left\{\varphi_{0}, \vartheta_{0}\right\}$, only. For notational brevity, we omit subscripts from the steering vector.

$$
\text { Since } \hat{\boldsymbol{Q}}_{\mathrm{n}}^{\mathrm{H}}\left(\mathrm{e}^{\mathrm{j} \Omega_{0}}\right) \hat{\boldsymbol{Q}}_{\mathrm{n}}\left(\mathrm{e}^{\mathrm{j} \Omega_{0}}\right)=\mathbf{I} \in \mathbb{R}^{K \times K} \text { (14) becomes }
$$

$$
\begin{aligned}
& \hat{\xi}_{\text {MUSIC }}^{-1}\left(\mathrm{e}^{\mathrm{j} \Omega_{0}}, \varphi_{0}, \vartheta_{0}\right)= \mathbf{a}^{\mathrm{H}}\left(\mathrm{e}^{\mathrm{j} \Omega_{0}}\right) \hat{\boldsymbol{Q}}_{\mathrm{n}}\left(\mathrm{e}^{\mathrm{j} \Omega_{0}}\right) \hat{\boldsymbol{Q}}_{\mathrm{n}}^{\mathrm{H}}\left(\mathrm{e}^{\mathrm{j} \Omega_{0}}\right) . \\
& \cdot \hat{\boldsymbol{Q}}_{\mathrm{n}}\left(\mathrm{e}^{\mathrm{j} \Omega_{0}}\right) \hat{\boldsymbol{Q}}_{\mathrm{n}}^{\mathrm{H}}\left(\mathrm{e}^{\mathrm{j} \Omega_{0}}\right) \mathbf{a}\left(\mathrm{e}^{\mathrm{j} \Omega_{0}}\right) \\
&=\left\|\hat{\boldsymbol{Q}}_{\mathrm{n}}\left(\mathrm{e}^{\mathrm{j} \Omega_{0}}\right) \hat{\boldsymbol{Q}}_{\mathrm{n}}^{\mathrm{H}}\left(\mathrm{e}^{\mathrm{j} \Omega_{0}}\right) \mathbf{a}\left(\mathrm{e}^{\mathrm{j} \Omega_{0}}\right)\right\|_{2}^{2} .
\end{aligned}
$$

Since $\boldsymbol{Q}_{\mathrm{n}}^{\mathrm{H}}\left(\mathrm{e}^{\mathrm{j} \Omega_{0}}\right) \mathbf{a}\left(\mathrm{e}^{\mathrm{j} \Omega_{0}}\right)=\mathbf{0}$, we can write (15) such that

$$
\begin{aligned}
\hat{\boldsymbol{\xi}}_{\text {MUSIC }}^{-1}\left(\mathrm{e}^{\mathrm{j} \Omega_{0}}, \varphi_{0}, \vartheta_{0}\right)= & \| \hat{\boldsymbol{Q}}_{\mathrm{n}}\left(\mathrm{e}^{\mathrm{j} \Omega_{0}}\right) \hat{\boldsymbol{Q}}_{\mathrm{n}}^{\mathrm{H}}\left(\mathrm{e}^{\mathrm{j} \Omega_{0}}\right) \mathbf{a}\left(\mathrm{e}^{\mathrm{j} \Omega_{0}}\right)- \\
& -\boldsymbol{Q}_{\mathrm{n}}\left(\mathrm{e}^{\mathrm{j} \Omega_{0}}\right) \boldsymbol{Q}_{\mathrm{n}}^{\mathrm{H}}\left(\mathrm{e}^{\mathrm{j} \Omega_{0}}\right) \mathbf{a}\left(\mathrm{e}^{\mathrm{j} \Omega_{0}}\right) \|_{2}^{2} \\
\leq & \left\|\hat{\boldsymbol{P}}_{\mathrm{n}}\left(\mathrm{e}^{\mathrm{j} \Omega_{0}}\right)-\boldsymbol{P}_{\mathrm{n}}\left(\mathrm{e}^{\mathrm{j} \Omega_{0}}\right)\right\|_{2}^{2}\left\|\mathbf{a}\left(\mathrm{e}^{\mathrm{j} \Omega_{0}}\right)\right\|_{2}^{2} \\
= & \operatorname{dist}\left\{\hat{\mathcal{U}}_{\mathrm{n}}\left(\mathrm{e}^{\mathrm{j} \Omega_{0}}\right), \mathcal{U}_{\mathrm{n}}\left(\mathrm{e}^{\mathrm{j} \Omega_{0}}\right)\right\}^{2} .
\end{aligned}
$$

Here we have that $\left\|\mathbf{a}\left(\mathrm{e}^{\mathrm{j} \Omega_{0}}\right)\right\|_{2}=1$ by design and using (9) with $\hat{\mathcal{U}}_{\mathrm{n}}\left(\mathrm{e}^{\mathrm{j} \Omega_{0}}\right)$ and $\mathcal{U}_{\mathrm{n}}\left(\mathrm{e}^{\mathrm{j} \Omega_{0}}\right)$.

Using (12), we can relate $\hat{\xi}_{\text {MUSIC }}^{-1}\left(\mathrm{e}^{\mathrm{j} \Omega_{0}}, \varphi_{0}, \vartheta_{0}\right)$ to the error matrix in (13) and the eigenvalue distance, $\delta$, in (10) such that

$$
\hat{\xi}_{\text {MUSIC }}^{-1}\left(\mathrm{e}^{\mathrm{j} \Omega_{0}}, \varphi_{0}, \vartheta_{0}\right) \leq \frac{16}{\delta^{2}}\left\|\mathbf{E}_{\mathrm{sn}}\left(\mathrm{e}^{\mathrm{j} \Omega_{0}}\right)\right\|_{2}^{2},
$$

whenever (11) is satisfied. Thus we can link the inverse MUSIC spectrum at the source AoA to the derived eigenspace perturbation in (16) and to its bound via (17).

\section{Results And Simulations}

\section{A. Scenario}

As an example, we look at the case of a single source ( $K=1$ ) impinging on an array of $M=3$ sensors from an angle of arrival of $\varphi_{0}=-60^{\circ}$ and no elevation i.e. $\vartheta_{0}=0^{\circ}$. The innovation filter $b_{1}[n]$ of Sec. II-A has a passband response, where the normalised passband frequencies are defined as $\Omega_{\text {pass }}=\left[\begin{array}{ll}0.2 \pi & 0.9 \pi\end{array}\right]$. This filter is excited by a zero-mean unit-variance uncorrelated complex circularly symmetric Gaussian source. The sample space-time covariance matrix, $\hat{\mathbf{R}}[\tau]$, is estimated from $N$ snapshots of data $\mathbf{x}[n]$ for 
$n=0, \ldots, N-1$ samples and with the support value, $\tau$, adjusted to be optimal [4]. For the simulations below, we vary the sample size from 200 to 10000 samples in intervals of 100 samples. Each parameter setting is tested over an ensemble of $10^{4}$ simulations so that we can analyse the statistics of the results. We consider only one fixed frequency at $\Omega_{0}=\frac{\pi}{4}$.

\section{B. MUSIC and Subspace Perturbations}

For the following results, we are interested in how the quantities in (11) and (17) perform, and investigate the validity of these for different $N$. For clarity, we convert (17) into a ratio such that

$$
\gamma\left(\mathrm{e}^{\mathrm{j} \Omega_{0}}\right)=\hat{\xi}_{\text {MUSIC }}^{-1}\left(\mathrm{e}^{\mathrm{j} \Omega_{0}}, \varphi_{0}, \vartheta_{0}\right) / \frac{16}{\delta^{2}}\left\|\mathbf{E}_{\mathrm{sn}}\left(\mathrm{e}^{\mathrm{j} \Omega_{0}}\right)\right\|_{2}^{2},
$$

which satisfies $\gamma\left(\mathrm{e}^{\mathrm{j} \Omega_{0}}\right) \leq 1$ if (11) is satisfied.

As discussed previously, the subspace distance is related to a condition specified in (11). We first look at the proportion of total simulations that satisfy (11) as a function of sample size; this is shown in Fig. 1 where we see this proportion increase as the sample size, $N$, increases which is to be expected. From this, it is important to note that often for smaller sample sizes we fail to satisfy the condition for the simulations carried out in this paper. We denote the simulations that pass this condition by the $\{\cdot\}^{\text {(pass) }}$ superscript.

In Fig. 2 we investigate the distribution of (18) for the simulations that pass (11) and from this result we verify that if (11) is satisfied then (17) is always satisfied. Since we often do not satisfy the condition in (11) we look at the distribution of $\gamma\left(\mathrm{e}^{\mathrm{j} \Omega_{0}}\right)$ for all simulations to see the overall performance of (18) and, therefore, test if (17) is useful independently of the condition. The result from Fig. 3 shows that as we increase $N$ more simulations satisfy these bounds, with the median of $\gamma\left(\mathrm{e}^{\mathrm{j} \Omega_{0}}\right)$ almost constant. For the curve given by the 95 th percentile, it can be seen that at $N \geq 400$ samples $\gamma\left(\mathrm{e}^{\mathrm{j} \Omega_{0}}\right)$ crosses $10^{0}$ and satisfies (17) and (18) for at least $95 \%$ of simulation runs. For $N<400$ we notice that for many of the trials, the 95th percentile curve is relatively close to one.

In Fig. 4 it can be seen that even though the distribution in Fig. 3 has 95th percentiles less than one, we still have a small percentage of simulations that fail to satisfy (17) for small sample sizes. As we increase the sample size to $N=2000$ this percentage tends to 0 showing that all simulations satisfy the bound irregardless of the condition in (11).

\section{Extraction of the Angle of Arrival}

In Sec. IV-B, we were concerned by how $\hat{\xi}_{\text {MUSIC }}^{-1}\left(\mathrm{e}^{\mathrm{j} \Omega_{0}}, \varphi_{0}, \vartheta_{0}\right)$ is affected at the ideal/expected angle of arrival. We now investigate the extraction of the angle of arrival from the inverse MUSIC spectra based on estimates.

If estimated quantities are used then the inverse MUSIC spectrum can vary and where we would expect the angle of arrival to be extracted i.e. at the global minima of $\hat{\xi}_{\text {MUSIC }}^{-1}\left(\mathrm{e}^{\mathrm{j} \Omega_{0}}, \varphi_{0}, \vartheta_{0}\right)$, could shift. However, as we have discussed, the use of more data can result in more accurate estimates. In this example, we extract an angle of arrival,

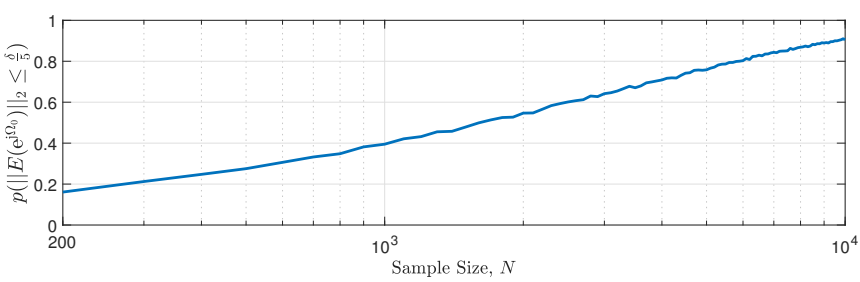

Fig. 1. Proportion of simulations that satisfy (11).

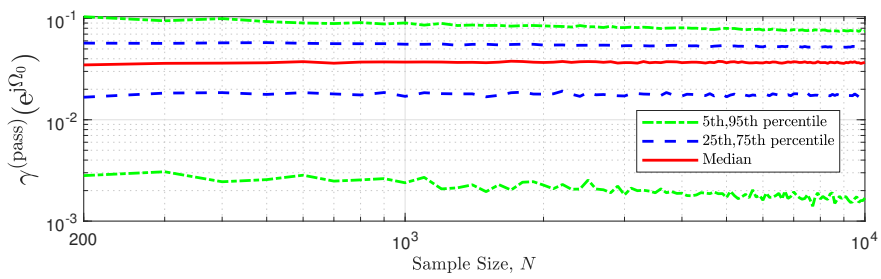

Fig. 2. Distribution of (18) for simulations that satisfy (11) with 5, 25, 75, and 95 th percentiles, where the median is shown by the solid red line.

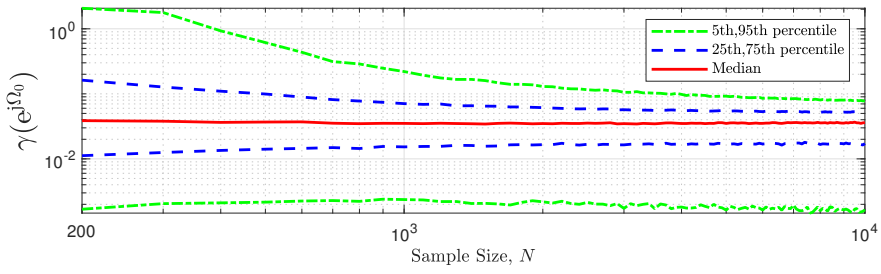

Fig. 3. Distribution of (18) for all ensemble probes with 5, 25, 75, and 95th percentiles, where the median is shown by the solid red line.

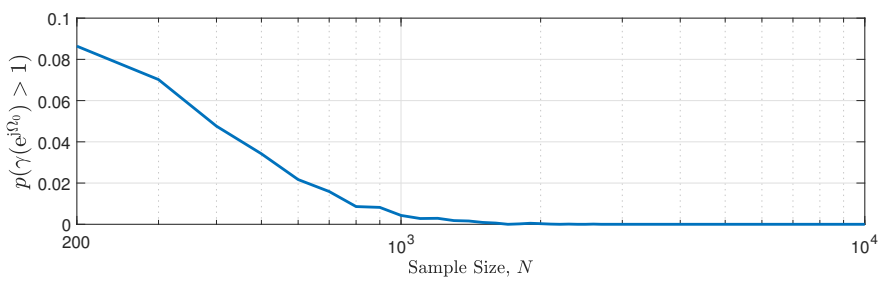

Fig. 4. Proportion of all ensemble probes that satisfy the ratio given in (18).

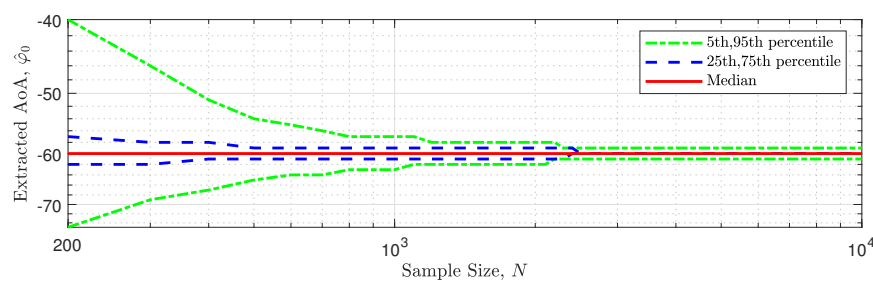

Fig. 5. Distribution of the extracted AoA, $\hat{\varphi}_{0}$, from estimated MUSIC spectra with $5,25,75$, and 95 th percentiles, where the median is shown by the solid red line.

$\hat{\varphi}_{0}=\min \left\{\hat{\xi}_{\text {MUSIC }}^{-1}\left(\mathrm{e}^{\mathrm{j} \Omega_{0}}, \varphi_{0}, \vartheta_{0}\right)\right\}$ from each inverse MUSIC spectra calculated from each simulation and show the results as a statistical distribution.

It can be seen from Fig. 5 that there is a large deviation at low sample sizes, so that if the global minimum was used then we could extract an angle of arrival that is far from the correct value. As we increase the number of samples then we are more likely to extract values closer to the true value with minimal variation. The median of the distribution is constant 


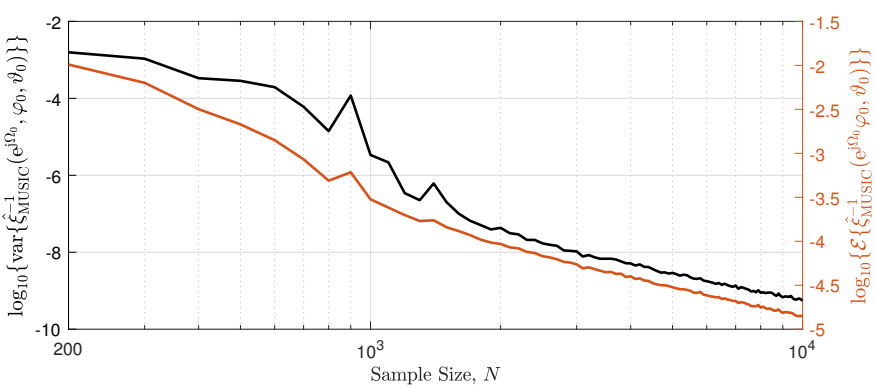

Fig. 6. Ensemble average (right) and variance (left) of $\hat{\xi}_{\mathrm{MUSIC}}^{-1}\left(\mathrm{e}^{\mathrm{j} \Omega_{0}}, \varphi_{0}, \vartheta_{0}\right)$ as a function of $N$ on a logarithmic scale.

throughout the large number of simulations and indicates that we can extract the correct estimate but not consistently as the percentiles demonstrate. In defense applications, it may be useful to track targets within a certain angular range. The results in Fig. 5 shows that this could be applied for more targets/sources or for a lower sample size if the restriction of a single accurate AoA was lifted. For the scenario in this paper, a sample size $N<200$ showed a heavy degradation in the extracted angle to the point that this angle can be ambiguous.

\section{Mean and Variance of MUSIC}

Given that we have discussed bounds and conditions related to the estimated MUSIC metric, $\hat{\xi}_{\text {MUSIC }}^{-1}\left(\mathrm{e}^{\mathrm{j} \Omega_{0}}, \varphi_{0}, \vartheta_{0}\right)$, we now look at this metric directly. If $\hat{\xi}_{\text {MUSIC }}^{-1}\left(\mathrm{e}^{\mathrm{j} \Omega_{0}}, \varphi_{0}, \vartheta_{0}\right)$ is evaluated at the ideal angle of arrival then we would expect that this value should be 0 . Due to the estimated quantities we would now expect $\hat{\xi}_{\text {MUSIC }}^{-1}\left(\mathrm{e}^{\mathrm{j} \Omega_{0}}, \varphi_{0}, \vartheta_{0}\right) \rightarrow 0$ as $N \rightarrow \infty$. Therefore, if a distribution of this function was calculated for all simulations then the mean and variance of this function should decrease as $N$ increases i.e. we should increase the reliability of $\hat{\xi}_{\text {MUSIC }}^{-1}\left(\mathrm{e}^{\mathrm{j} \Omega_{0}}, \varphi_{0}, \vartheta_{0}\right)$ as we increase $N$.

As demonstrated in Fig. 6 we notice that both the ensemble average and variance decrease on a logarithmic scale. This, combined with Fig. 5, allows us to see that the reliability of the inverse MUSIC algorithm estimated from quantities calculated from finite data increases as we use more data.

\section{CONCLUSION}

When estimating a space-time covariance matrix, it is imperative to understand the effect of perturbations before using real-world applications such as the MUSIC algorithm. In this paper we have: (i) discussed how eigenspaces can be perturbed as a result of using space-time covariance matrices estimated from limited data, (ii) stated theoretical bounds for the perturbations of subspaces, and (iii) provided a condition which depends on the error between ideal and estimated spacetime covariance matrices and the distance between signal-plusnoise and noise-only eigenvalues for the ideal case.

We have shown that subspace perturbations can be used to derive a theoretical bound to describe the degradation of the MUSIC algorithm, evaluated at a single frequency point, as a function of the sample size used. We tested the performance of this bound as well as the condition on which it depends.
For the simulations carried out it can be verified that if the condition is satisfied then this bound is always satisfied for all sample sizes tested. If this condition is not satisfied e.g. for low sample sizes, then it can be shown that the bound is satisfied $75 \%$ of the time.

If we now relate these findings to the extraction of an AoA then this can be shown to vary as a function of the sample size. Given a large number of samples we can successfully extract the AoA with small statistical variation. Additionally as we increase the number of samples, the extracted AoA tends towards the ideal case with decreasing variance.

\section{REFERENCES}

[1] X. Mestre, "Improved estimation of eigenvalues and eigenvectors of covariance matrices using their sample estimates," IEEE Trans. Information Theory, vol. 54, no. 11, pp. 5113-5129, Nov 2008.

[2] C. Delaosa, F. K. Coutts, J. Pestana, and S. Weiss, "Impact of space-time covariance estimation errors on a parahermitian matrix EVD," in IEEE SAM, Sheffield, UK, July, July 2018, pp. 1-5. [Online]. Available: https://strathprints.strath.ac.uk/63998/

[3] X. Mestre, "On the asymptotic behavior of the sample estimates of eigenvalues and eigenvectors of covariance matrices," IEEE Trans. Signal Processing, vol. 56, no. 11, pp. 5353-5368, 2008.

[4] C. Delaosa, J. Pestana, N. J. Goddard, S. D. Somasundaram, and S. Weiss, "Support estimation of a sample space-time covariance matrix," in SSPD, Brighton, UK, May, May 2019, pp. 1-5.

[5] R. O. Schmidt, "Multiple emitter location and signal parameter estimation," vol. 34, no. 3, pp. 276-280, March 1986.

[6] G. H. Golub and C. F. Van Loan, Matrix Computations, 4th ed Baltimore, Maryland: John Hopkins University Press, 2013.

[7] J. K. Thomas, L. L. Scharf, and D. W. Tufts, "The probability of a subspace swap in the SVD," IEEE Trans. Signal Processing, vol. 43, no. 3, pp. 730-736, 1995.

[8] M. Shaghaghi and S. A. Vorobyov, "Subspace leakage analysis and improved DOA estimation with small sample size," IEEE Trans. Signal Processing, vol. 63, pp. 3251-3265, 2015.

[9] E. Ollila and V. Koivunen, "Robust antenna array processing using mestimators of pseudo-covariance," in IEEE PIMRC, vol. 3:2659-2663, Beijing, China, Sep. 2003.

[10] X. Mestre and F. Rubio, "An improved subspace-based algorithm in the small sample size regime," in IEEE ICASSP, vol. 4:1073-1076, Toulouse, France, 2006.

[11] M. Shaghaghi and S. A. Vorobyov, "Iterative root-music algorithm for DOA estimation," in IEEE CAMSAP, St. Martin, France, Dec 2013, pp. 53-56.

[12] A. Kangas, P. Stoica, and T. Soderstrom, "Finite sample and modelling error effects on esprit and music direction estimators," IEE Proceedings - Radar, Sonar and Navigation, vol. 141, no. 5, pp. 249-255, 1994.

[13] T. I. Laakso, V. Välimäki, M. Karjalainen, and U. K. Laine, "Splitting the Unit Delay," IEEE Signal Processing Magazine, vol. 13:30-60, January 1996.

[14] J. Selva, "An efficient structure for the design of variable fractional delay filters based on the windowing method," IEEE Trans. Signal Processing, vol. 56:3770-3775, Aug. 2008.

[15] S. Weiss, J. Pestana, and I. K. Proudler, "On the existence and uniqueness of the eigenvalue decomposition of a parahermitian matrix," IEEE Transactions on Signal Processing, vol. 66, no. 10, pp. 2659-2672, May 2018.

[16] S. Weiss, J. Pestana, I. K. Proudler, and F. K. Coutts, "Corrections to "on the existence and uniqueness of the eigenvalue decomposition of a parahermitian matrix"," IEEE Trans. Signal Processing, vol. 66, no. 23, pp. 6325-6327, May 2018.

[17] S. Weiss, I. K. Proudler, F. K. Coutts, and J. Pestana, "Iterative approximation of analytic eigenvalues of a parahermitian matrix EVD," in IEEE ICASSP, Brighton, UK, May 2019, pp. 8038-8042.

[18] A. Papoulis, Probability, Random Variables, and Stochastic Processes, 3rd ed. New York: McGraw-Hill, 1991.

[19] C. Delaosa, J. Pestana, N. J. Goddard, S. Somasundaram, and S. Weiss, "Sample space-time covariance matrix estimation," in IEEE ICASSP, Brighton, UK, 2019, pp. 8033-8037. 